\title{
Application of Higher Order Haar Wavelet Method for Solving Nonlinear Evolution Equations
}

\author{
Mart Ratas and Andrus Salupere \\ Department of Cybernetics, School of Science, Tallinn University of \\ Technology \\ 12618 Tallinn, Estonia \\ E-mail(corresp.): mart.ratas@ttu.ee
}

Received August 29, 2019; revised February 3, 2020; accepted February 7, 2020

\begin{abstract}
The recently introduced higher order Haar wavelet method is treated for solving evolution equations. The wave equation, the Burgers' equations and the Korteweg-de Vries equation are considered as model problems. The detailed analysis of the accuracy of the Haar wavelet method and the higher order Haar wavelet method is performed. The obtained results are validated against the exact solutions.
\end{abstract}

Keywords: Haar wavelets, evolution equations, higher order wavelet expansion.

AMS Subject Classification: 37M05; 65T60; 35Q51.

\section{Introduction}

From the mathematical viewpoint evolution equations under consideration are partial differential equations (PDEs). In case of numerical solution of PDEs one has to approximate the partial derivatives with respect to time and space coordinates. Local or global methods can be used for these approximations. The finite difference method is the most typical example of local methods. In the case of global methods, usually the function itself is approximated as a sum of basis functions (for example trigonometric functions). However, trigonometric functions are not the only choice for basis functions. For example, in [64] mixed Laguerre-Legendre interpolation approximation is applied. One of the more popular global methods is the fast Fourier transform (FFT) related pseudospectral method $[20,22,55,56,58,59]$. In the present paper another global approach is applied: Haar wavelets are used for approximation of partial derivatives. 
Recently, the higher order Haar wavelet method (HOHWM) has been developed by Majak et. al. [42] as an improvement of the Haar wavelet method (HWM) originally introduced by Chen and Hsiao in [15]. The HWM has been proposed for solving differential equations $[15,27,51]$ as well as a wide class of integro-differential and integral equations [5, 7, 8, 14, 35, 37, 63]. According to HWM, as proposed in [15,27], the highest order derivative included in the differential equation is expanded into the series of Haar functions. This approach is based on the fact that the Haar functions are given in form of quadratic waves which are not differentiable. A different approach was introduced in [13], according to which the quadratic waves can be regularized. However, the latter approach appears more complicated and has not found wider use. In addition to the apparant simpler implementation, the former approach has commonly been utilized in studies related to the development and application of HWM. In pioneering works by Lepik $[33,34,35,36,37,38]$, integration techniques for HWM were developed. A thorough overview of HWM and its application in different research areas can be found in the monograph [39]. The weak formulation based HWM was introduced by Majak et. al. in [41]. The complexity issues of strong and weak formulation based HWM are also discussed in the latter study.

One of the most common areas of application for HWM appears to be engineering. This specific field includes solid mechanics [35,36,38], composites $[26,28,40,66,68,69]$, etc. Additionally, free vibration analysis of the multilayer composite plate was performed in [40] and in $[28,66,67,68,69]$ the HWM was adopted for free vibration analysis of composite laminated conical and cylindrical shell, and annular plate structures.

The fractional differential and integro-differential equations form a challenging application area for new numerical methods. This also applies to HWM. Due to their specific nature, less coverage by numerical methods and commercial software has been observed. In a pioneering study the Caputo derivatives are utilized and the differential equations are converted to integral equations including integer order derivatives [37]. Thus, the wavelet expansion is applied to derivatives of integer order. An alternate approach was employed in $[44,53,60,62]$ where the fractional derivatives are expanded directly to wavelet series.

Recent developments in this area have lead to HWM being treated for multidimensional case $[3,4,6]$ as well an adaptive wavelet method [23]. Other devolopments include nonuniform HWM [48], application in inverse problems [21], identification of software piracy [47], etc.

The key factors of any numerical method are the accuracy and convergence. These are studied for HWM in $[43,45,65]$. The convergence theorem is proven in [45], which also shows that the order of convergence of the HWM approach based on [15] is equal to two. The latter result gives an incentive to further improve HWM. In the comparative study [30] it was shown that HWM proposed in $[15,27]$ is not competitive with other simple strong formulation based methods used in engineering like differential quadrature method and finite difference method. Motivated by the latter conclusions Majak et.al. developed HOHWM in [42]. 
In the current study the HOHWM is treated for solving evolution equations. In addition, the wave equation was chosen as the first model equation for its simplicity. The Burgers' equation $[10,11]$ has previosuly been studied using the HWM $[29,36,41,49]$ and also has an anlytical solution, thus the possibility of comparison made it a decent choice for a model equation. It finds applications in modeling of turbulence [10] and traffic [46], as well as in weak non-stationary shock wave in real fluids [31] and in nonlinear acoustics [24]. The HWM has also previously been applied to the Korteweg-de Vries (KdV) equation in [50, 52]. The KdV equation was derived in order to describe the movement of long unidirectional shallow water waves in a rectangular channel but has been found to model different nonlinear phenomena nowadays [18,19,54]. A detailed analysis of the improved accuracy of HOHWM over HWM is performed for all three model equations.

The article is structured as follows. Firstly, the Haar wavelet family is introduced in Section 2. Secondly, the model equations used are described in Section 3. Section 4 outlines the HWM and HOHWM. The analysis of the results is presented in Section 5 and conclusions are drawn in the final section.

\section{Haar wavelet family}

In the following the Haar wavelet family is defined utilizing the notation introduced by Lepik in [38]. $2 M$ subintervals of equal length $\Delta x=(B-A) /(2 M)$ form the integration domain $x \in[A, B]$. The maximum level of resolution $J$ is defined as $J=\log _{2}(M)$. The Haar wavelet family for a fixed $M$ can be described as

$$
h_{i}(x)= \begin{cases}1, & \text { for } \quad x \in\left[\xi_{1}(i), \xi_{2}(i)\right) \\ -1, & \text { for } x \in\left[\xi_{2}(i), \xi_{3}(i)\right) \\ 0, & \text { elsewhere }\end{cases}
$$

where

$$
\begin{aligned}
& \xi_{1}(i)=A+2 k \mu \Delta x, \quad \xi_{2}(i)=A+(2 k+1) \mu \Delta x, \quad \xi_{3}(i)=A+2(k+1) \mu \Delta x, \\
& \mu=M / m, \quad m=2^{j}, \quad \Delta x=(B-A) /(2 M) .
\end{aligned}
$$

In $(2.1)$ and $(2.2) j=0,1, \ldots, J$ is the dilation parameter, $k=0,1, \ldots, m-1$ is translation parameter, $m$ corresponds to the maximum number of square waves in the interval $x \in[A, B]$ for the given dilation and index $i$ is calculated from $i=m+k+1$. Therefore (2.2) corresponds to $i \geq 2$ since $m \geq 1$ and $k \geq 0$. The scaling function $h_{1}(x)=1$ is constant, in this case the values $m=0, \xi_{1}=A, \xi_{2}=B, \xi_{3}=B$ are considered. The other Haar functions contain a single square wave each. The Haar functions are orthogonal to each other and therefore form a good transform basis

$$
\int_{A}^{B} h_{i}(x) h_{l}(x) d x= \begin{cases}2^{-j}, & i=l=2^{j}+k \\ 0, & i \neq l\end{cases}
$$


Thus, any square integrable function $f(x)$ can be expanded into Haar wavelets as

$$
f(x)=\sum_{i=1}^{\infty} a_{i} h_{i}(x)
$$

where $a_{i}$ denote the Haar coefficients.

According to [38] the integrals of the Haar functions (2.1) of order $n$ can be calcualted analytically as follows

$$
p_{n, i}(x)=\left\{\begin{array}{l}
0, \quad \text { for } \quad x \in\left[A, \xi_{1}(i)\right), \\
\frac{\left(x-\xi_{1}(i)\right)^{n}}{n !}, \text { for } x \in\left[\xi_{1}(i), \xi_{2}(i)\right), \\
\frac{\left(x-\xi_{1}(i)\right)^{n}-2\left(x-\xi_{2}(i)\right)^{n}}{n !}, \text { for } x \in\left[\xi_{2}(i), \xi_{3}(i)\right), \\
\frac{\left(x-\xi_{1}(i)\right)^{n}-2\left(x-\xi_{2}(i)\right)^{n}+\left(x-\xi_{3}(i)\right)^{n}}{n !}, \text { for } x \in\left[\xi_{3}(i), B\right) .
\end{array}\right.
$$

Within the present paper the matrix form of the above formulation is used. Therefore, the elements of $(2 M) \times(2 M)$ matrix $\mathbf{H}$, are given as values of the Haar functions

$$
H_{i l}=h_{i}\left(x_{l}\right)
$$

at collocation points $x_{l}=(l-1 / 2) \Delta x$. The $(2 M) \times(2 M)$ matrix $\mathbf{P}_{n}$ with elements

$$
\left(P_{n}\right)_{i l}=p_{n, i}\left(x_{l}\right),
$$

denotes the $n$th integral of the Haar wavelet matrix for a given resolution $J$.

Using (2.5) and (2.6) and considering the coefficient vector a one obtains $\mathbf{f}(\mathbf{x})=\mathbf{a} \cdot \mathbf{H}$ instead of (2.3) and

$$
\underbrace{\int \cdots \int_{A}^{x}}_{n} \mathbf{H} \underbrace{d \xi \ldots d \xi}_{n}=\mathbf{P}_{n} .
$$

It must be noted, that the matrices $\mathbf{H}$ and $\mathbf{P}_{n}$ depend on vector $\mathbf{x}$ of collocation points. The statements in (2.4) imply that in boundary points $A$ and $B$ hold

$$
\begin{aligned}
& \left(P_{n}(A)\right)_{i}=0, \quad \forall n>0, \quad \forall i, \\
& \left(P_{n}(B)\right)_{i}=p_{n, i}(B)=\frac{\left(B-\xi_{1}(i)\right)^{n}-2\left(B-\xi_{2}(i)\right)^{n}+\left(B-\xi_{3}(i)\right)^{n}}{n !} .
\end{aligned}
$$

$\mathbf{P}_{n}(0)$ and $\mathbf{P}_{n}(B)$ form column vectors which will be used when forming a system of equations that satsify the boundary conditions. These expressions can often be simplified due to equation (2.7).

\section{Model equations}

The wave equation

$$
u_{t t}=c^{2} u_{x x},
$$

subject to the initial and boundary conditions

$$
u(x, 0)=u_{0}(x), \quad u_{t}(x, 0)=v_{0}(x), \quad u(A, t)=u_{l}(t), \quad u(B, t)=u_{r}(t),
$$


was considered as the first model equation. The particular exact solution used was a travelling wave solution of the form

$$
u(x, t)=\left(1+e^{c_{1}\left(x-c t-x_{0}\right)}\right)^{-1}
$$

where $c_{1}$ is a parameter that varies the steepness of the shockwave, $c$ is its travelling speed and $x_{0}$ its initial phase.

As the second model equation, the Burgers' equation

$$
u_{t}+u u_{x}=\nu u_{x x}
$$

was chosen. Here $\nu$ is the diffusion coefficient. The Burgers' equation subjected to the initial and boundary conditions

$$
u(x, 0)=u_{0}(x), \quad u(A, t)=u_{l}(t), \quad u(B, t)=u_{r}(t)
$$

was considered. Its analytical solution has been found in the form of

$$
u(x, t)=\frac{2 \nu \pi}{L} \frac{\sum_{n=1}^{\infty} \exp \left(-E_{n} t\right) n I_{n}\left(R_{0} /(2 \pi)\right) \sin (n \pi x / L)}{I_{0}\left(R_{0} /(2 \pi)\right)+2 \sum_{n=1}^{\infty} \exp \left(-E_{n} t\right) I_{n}\left(R_{0} /(2 \pi)\right) \cos (n \pi x / L)},
$$

where $R_{0}=u_{0} L / \nu$ is the Reynolds number, $E_{n}=\nu n^{2} \pi^{2} / L^{2}, I_{n}$ represents the modified Bessel functions of first kind and $L=B-A$ is the $x$ domain range $[9,16]$.

The third model equation was chosen to be the $\mathrm{KdV}$ equation of the form

$$
u_{t}+\alpha u u_{x}+u_{x x x}=0
$$

subjected to the initial and boundary conditions

$$
u(x, 0)=u_{0}(x), \quad u(A, t)=u_{l}(t), \quad u(B, t)=u_{r}(t), \quad u_{x}(A, t)=u_{l, x}(t) .
$$

An exact one soliton solution for the $\mathrm{KdV}$ equation (3.5) is known in the form of

$$
u(x, t)=\frac{3 c}{\alpha} \operatorname{sech}^{2}\left(\frac{1}{2} \sqrt{c}\left(x-c t-x_{0}\right)\right),
$$

where $c$ is the speed of the travelling soliton, $\alpha$ is the nonlinear coefficient in the equation and $x_{0}$ denotes the initial phase. There exists also a two-soliton solution $[1,32,57,70]$ in the form

$$
u(x, t)=\frac{3\left(c_{B}-c_{S}\right)}{|\alpha|} \frac{\left(c_{B} \operatorname{csch}^{2}\left(\xi_{B}\right)+c_{S} \operatorname{sech}^{2}\left(\xi_{S}\right)\right)}{\left(\sqrt{c_{B}} \operatorname{coth}\left(\xi_{B}\right)-\sqrt{c_{S}} \tanh \left(\xi_{S}\right)\right)^{2}},
$$

where $\xi_{B}=\frac{1}{2} \sqrt{c_{B}}\left(x-c_{B} t-x_{0 B}\right), \xi_{S}=\frac{1}{2} \sqrt{c_{S}}\left(x-c_{S} t-x_{0 S}\right)$, and $c_{B}$ and $c_{S}$ are the speeds of the bigger and smaller soliton, respectively, $x_{0 B}$ and $x_{0 S}$ are the initial phases for the bigger and smaller soliton, respectively and $\alpha$ is the nonlinear parameter. Using (3.7) soliton interactions can be observed. 


\section{Haar wavelet methods}

In many numerical studies $[2,12,25,41]$, spatial derivatives are expanded into Haar wavelet series while finite difference type schemes are used for integration with respect to time. In the present study, MATLAB's ode45 [61] solver based on Runge-Kutta $(4,5)$ formula [17] is used for integration with respect to time. This allows one to calculate the value of the function at each time moment from its values at previous time moments without the need to expand both axes into the Haar series. The latter often results in extremely large matrices which can get computationally expensive.

The well known HWM involves expanding in the Haar wavelet series the highest order derivative present within the equation. One then obtains for the $n$th spatial derivative

$$
\mathbf{u}_{n x}(\mathbf{x})=\mathbf{a} \cdot \mathbf{H}_{x},
$$

where the subscript notes the axis along which the HWM (as well as the HOHWM) is deployed and $\mathbf{a}$ is the Haar wavelet coefficient vector. After integrating $n$ times one arrives at the function itself as

$$
\mathbf{u}(\mathbf{x})=\mathbf{a} \cdot \mathbf{P}_{x n}+\sum_{i=0}^{n-1}\left(c_{i} \frac{\mathbf{x}^{i}}{i !}\right),
$$

where the unkown coefficients $c_{i}$ can be calculated by using the boundary conditions and $\mathbf{x}^{i}$ denotes the collocation vector with its elements raised to the power of $i$. The superscript of the vector of collocation points will refer to the element wise multiplication throughout the rest of the present study.

When it comes to the HOHWM, one needs to start with a derivative of a higher order than that which is present in the equation. In general, an additional $2 s$ derivatives are used. However, in the current study, only $s=1$ is considered. Thus, one starts with

$$
\mathbf{u}_{(n+2) x}(\mathbf{x})=\mathbf{a} \cdot \mathbf{H}_{x}
$$

and after integration arrives at

$$
\mathbf{u}(\mathbf{x})=\mathbf{a} \cdot \mathbf{P}_{x(n+2)}+\sum_{i=0}^{n+2}\left(c_{i} \frac{\mathbf{x}^{i}}{i !}\right),
$$

where two extra coefficients, $c_{n}$ and $c_{n+1}$ are introduced. In order to calculate those extra coefficients, some extra information is needed. In the present study the equation is evaluated at the boundary.

When the unkown coefficients $c_{i}$ have been obtained, the function $u$ can be described as

$$
\begin{aligned}
\mathbf{u}(\mathbf{x}, t)=\mathbf{a} \cdot \mathbf{R}_{x n}+\mathbf{S}_{x n}, & \text { for HWM, } \\
\mathbf{u}(\mathbf{x}, t)=\mathbf{a} \cdot \mathbf{R}_{x(n+2)}+\mathbf{S}_{x(n+2)}, & \text { for HOHWM, }
\end{aligned}
$$

where $\mathbf{R}_{x m}$ is a matrix and $\mathbf{S}_{x m}$ a vector obtained only from the Haar matrices and boundary conditions. They both depend on $x$. $\mathbf{S}_{x m}$ can also depend on $t$ 
(depending on the boundary conditions). Thus for the $k$ th derivative $(k<m)$ of $u$ one can write

$$
\begin{array}{cl}
\mathbf{u}_{k x}(\mathbf{x}, t)=\mathbf{a} \cdot \mathbf{R}_{x(n-k)}+\mathbf{S}_{x(n-k)}, & \text { for HWM, } \\
\mathbf{u}_{k x}(\mathbf{x}, t)=\mathbf{a} \cdot \mathbf{R}_{x(n+2-k)}+\mathbf{S}_{x(n+2-k)}, & \text { for HOHWM. }
\end{array}
$$

It must be noted that $\mathbf{R}_{x 0}=\mathbf{H}_{x}$ and $\mathbf{S}_{x 0}=0$ due to (4.1) and (4.2) in case of HWM. Once $\mathbf{R}_{x m}$ and $\mathbf{S}_{x m}$ have been found, one can arrive at

$$
\mathbf{a}=\left(u(x, t)-\mathbf{S}_{x m}\right) \cdot \mathbf{R}_{x m}^{-1},
$$

where $m=n$ (HWM) or $m=n+2$ (HOHWM). Equation (4.4) can be used to find the wavelet coefficient vector a from the previous iteration.

Finally, the equation in question will need to be arranged in the form $u_{t}=$ $f\left(u, u_{x}, u_{x x}, \ldots, u_{n x}\right)$ or $u_{t t}=f\left(u, u_{x}, u_{x x}, \ldots, u_{n x}\right)$. Then, (4.4) can be used to calculate a, after which (4.3) can be used to substitute $u$ and its derivatvies into the right hand side of the equation in question. This routine has to be followed for each time step.

In the following subsections, the numerical approach is described for each model equation. Firstly, the HWM is described. Then the extra conditions to find the extra coefficients added by HOHWM are introduced. Finally, HOHWM is described. The domain is fixed as $A=0$ and $B=1$.

\subsection{Wave equation}

Given constant boundary conditions

$$
u(0, t)=1, \quad u(1, t)=0,
$$

one obtains

$$
\mathbf{R}_{x 2}=\mathbf{P}_{x 2}-\mathbf{P}_{x 2}(1) \cdot \mathbf{x}, \quad \mathbf{S}_{x 2}=c_{l}(1-\mathbf{x})+c_{r} \mathbf{x}
$$

for HWM. For HOHWM the equation is evaluated at the boundary points as

$$
u_{t t}(0, t)=c^{2} u_{x x}(0, t), \quad u_{t t}(1, t)=c^{2} u_{x x}(1, t) .
$$

Since the boundary conditions (4.5) are constant in time $u_{t}(0, t)=u_{t}(1, t)=$ $u_{t t}(0, t)=u_{t t}(1, t)=0$. Thus one arrives at

$$
u_{x x}(0, t)=0, \quad u_{x x}(1, t)=0 .
$$

The addition of (4.7) gives

$$
\mathbf{R}_{x 4}=\mathbf{P}_{x 4}-\mathbf{P}_{x 4}(1) \cdot x-\mathbf{P}_{x 2}(1) \cdot\left(\mathbf{x}^{3}-1\right), \quad \mathbf{S}_{x 4}=1-\mathbf{x} .
$$

\subsection{Burgers' equation}

Given the homogeneous boundary conditions

$$
u(0, t)=0, \quad u(1, t)=0,
$$


one again obtains the same results (4.6) for HWM. However, for HOHWM the equation is again evaluated at the boundary points as

$$
\begin{aligned}
& u_{t}(0, t)+u(0, t) u_{x}(0, t)-\nu u_{x x}(0, t)=0, \\
& u_{t}(1, t)+u(1, t) u_{x}(1, t)-\nu u_{x x}(1, t)=0 .
\end{aligned}
$$

Since the boundary conditions (4.8) are homogeneous $u_{t}(0, t)=u_{t}(1, t)=0$ and (4.9) simplifies to

$$
u_{x x}(0, t)=0 \quad u_{x x}(1, t)=0 .
$$

The addition of (4.10) gives

$$
\mathbf{R}_{x 4}=\mathbf{P}_{x 4}-\mathbf{P}_{x 4}(1) \cdot \mathbf{x}+\mathbf{P}_{x 2}(1) \cdot \frac{\mathbf{x}-\mathbf{x}^{3}}{6}, \quad \mathbf{S}_{x 4}=0 .
$$

\subsection{KdV equation}

Given homogeneous boundary conditions

$$
u(0, t)=u(1, t)=u_{x}(1, t)=0,
$$

for HWM one obtains

$$
\mathbf{R}_{x 3}=\mathbf{P}_{x 3}+\mathbf{P}_{x 2}(1) \cdot\left(\mathbf{x}-\mathbf{x}^{2}\right)+\mathbf{P}_{x 3}(1) \cdot\left(\mathbf{x}^{2}-2 \mathbf{x}\right), \quad \mathbf{S}_{x 3}=0 .
$$

For HOHWM the equation is yet again evaluated at the boundary points as

$$
u_{t}(0, t)+\alpha u(0, t) u_{x}(0, t)+u_{x x x}(0, t)=0, u_{t}(1, t)+\alpha u(1, t) u_{x}(1, t)+u_{x x x}(1, t)=0 .
$$

Since the boundary conditions (4.11) are homogeneous $u_{t}(0, t)=u_{t}(1, t)=0$ and the above simplifies to

$$
u_{x x x}(0, t)=0, \quad u_{x x x}(1, t)=0 .
$$

The addition of (4.12) gives

$$
\begin{aligned}
\mathbf{R}_{x 5}= & \mathbf{P}_{x 5}+\frac{1}{2} \mathbf{P}_{x 3}(1) \cdot\left(-\mathbf{x}^{4}+2 \mathbf{x}^{3}-\mathbf{x}^{2}\right)+\frac{1}{2} \mathbf{P}_{x 4}(1) \cdot\left(4 \mathbf{x}^{4}-10 \mathbf{x}^{3}+6 \mathbf{x}^{2}\right) \\
& +\frac{1}{2} \mathbf{P}_{x 5}(1) \cdot\left(-6 \mathbf{x}^{4}+16 \mathbf{x}^{3}-12 \mathbf{x}^{2}\right) \\
\mathbf{S}_{x 5}= & 0 .
\end{aligned}
$$

\section{$5 \quad$ Numerical results}

Numerical experiments were carried out for equations (3.1), (3.3) and (3.5) with both HWM and HOHWM for different values of the resolution parameter $J$. The results were compared to the corresponding exact solutions $(3.2),(3.4)$ and (3.6), respectively. The maximal deviation of the calculated result with respect to the exact solution is traced. In case of the Burgers' equation, the time at which calculations become insufficiently accurate was measured in addition to 
the deviation. This is because given the near-singular behaviour of the exact solution at the boundary at the low values of $\nu$ used the integration scheme can become unstable. In case of the KdV equation, since the absolute value of the solution can be rather large, maximum relative deviation is traced in addition to maximum deviation. The results of HWM are compared with HOHWM.

Calculations for wave equation were carried out with initial condition from the exact solution (3.2) at $t=0$ as

$$
u_{0}(x)=\left(1+e^{c_{1}\left(x-x_{0}\right)}\right)^{-1}
$$

and the boundary conditions (4.5). In case of HOHWM the additional conditions (4.7) were added. The maximum deviation from the exact solution in case of $c=1 / 2$ and $x_{0}=1 / 4$ for $c_{1}=60,70,80$ within the domain $x \in[0,1]$, $t \in[0,1]$ can be seen in Table 1 .

Table 1. Results for the wave equation: maximal deviation of the numerical solution from the exact solution $\max \Delta u$ against the resolution parameter $J$ with initial conditions (5.1) and boundary conditions $(4.5)\left(c=1 / 2, x_{0}=1 / 4, x \in[0,1], t \in[0,1], c_{1}=60,70,80\right)$.

\begin{tabular}{cccccccc}
\hline \multirow{2}{*}{$J$} & $2 M$ & \multicolumn{6}{c}{$\max \Delta u$} \\
\cline { 3 - 8 } & & \multicolumn{2}{c}{$c_{1}=60$} & \multicolumn{2}{c}{$c_{1}=70$} & \multicolumn{2}{c}{$c_{1}=80$} \\
\cline { 3 - 8 } & & HWM & HOHWM & HWM & HOHWM & HWM & HOHWM \\
\hline 3 & 16 & 0.1336325 & 0.1106219 & 0.1799650 & 0.1614320 & 0.2428010 & 0.2203232 \\
4 & 32 & 0.1127782 & 0.0480090 & 0.1420468 & 0.0695581 & 0.1687752 & 0.0912491 \\
5 & 64 & 0.0522854 & 0.0077165 & 0.0744285 & 0.0138613 & 0.0987152 & 0.0220050 \\
6 & 128 & 0.0159480 & 0.0006604 & 0.0242365 & 0.0013906 & 0.0342071 & 0.0025845 \\
7 & 256 & 0.0042292 & 0.0000415 & 0.0066610 & 0.0000903 & 0.0098198 & 0.0001786 \\
\hline
\end{tabular}

Table 2. Results for the Burgers' equation: maximal time $t_{f}$ at which the numerical solutions deviates from the exact solution less than $10^{-3}$ against the resolution parameter $J$ with initial conditions (5.2) and boundary conditions (4.8) $(x \in[0,1], t \in[0,1], \nu=$ $\left.\frac{1}{100 \pi}, \frac{1}{110 \pi}, \frac{1}{120 \pi}\right)$.

\begin{tabular}{cccccccc}
\multirow{2}{*}{$J$} & $2 M$ & \multicolumn{6}{c}{$t_{f}$} \\
\cline { 3 - 8 } & & \multicolumn{2}{c}{$\nu=\frac{1}{100 \pi}$} & \multicolumn{2}{c}{$\nu=\frac{1}{110 \pi}$} & \multicolumn{2}{c}{$\nu=\frac{1}{120 \pi}$} \\
\cline { 3 - 8 } & HWM & HOHWM & HWM & HOHWM & HWM & HOHWM \\
\hline 3 & 16 & 0.1625 & 0.2375 & 0.1625 & 0.2375 & 0.1625 & 0.2375 \\
4 & 32 & 0.225 & 0.2875 & 0.225 & 0.275 & 0.225 & 0.275 \\
5 & 64 & 0.275 & 0.2875 & 0.275 & 0.3181 & 0.2625 & 0.3 \\
6 & 128 & 0.3106 & 0.3580 & 0.308 & 0.3542 & 0.3038 & 0.3475 \\
7 & 256 & 0.3400 & 0.5 & 0.3364 & 0.4646 & 0.3317 & 0.4143 \\
8 & 512 & 0.3801 & 0.5 & 0.3671 & 0.5 & 0.3657 & 0.5 \\
\hline
\end{tabular}

The Burgers' equation was solved for diffusion coefficient values of $\nu=$ $\frac{1}{100 \pi}, \frac{1}{110 \pi}, \frac{1}{120 \pi}$ within the domain $x \in[0,1], t \in\left[0, t_{f}\right]$. The initial condition

$$
u_{0}(x)=\sin (\pi x)
$$


and boundary conditions (4.8) were used. In case of HOHWM the additional conditions (4.10) were added. Due to the near-singular nature of the solution, the numerical solution can get unstable near the steep slope. Thus the time at which sufficient accuracy was lost $t_{f}^{n}$ is shown instead of the maximum error. The numerical result was taken to be sufficiently accurate if the maximum deviation with respect to the exact solution was smaller than $10^{-3}$. Corresponding results can be seen in Table 2. Figure 1 shows how the maximum error behaves in time for different resolutions at different values of $\nu$.
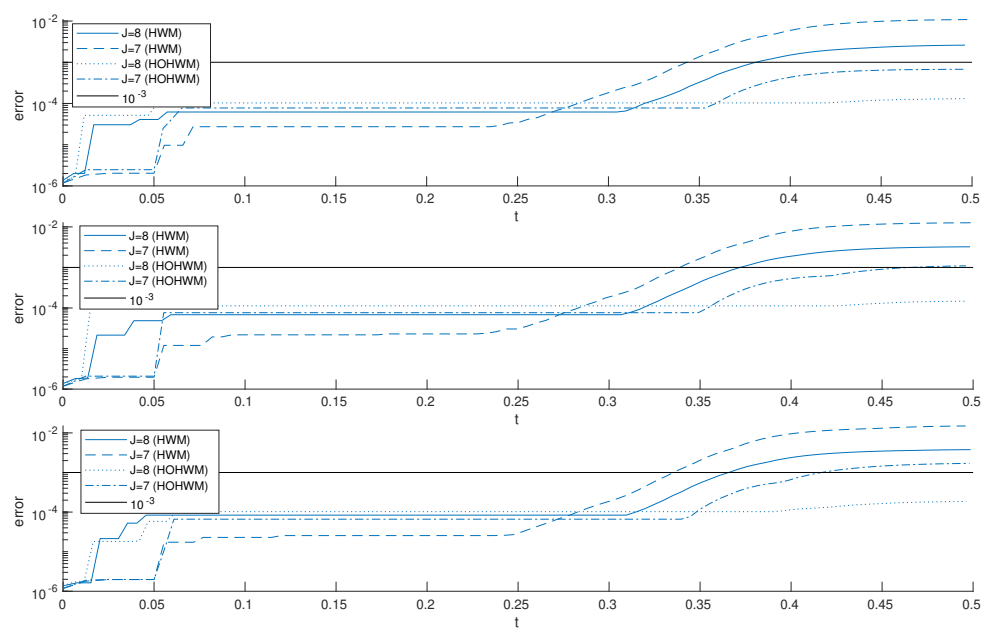

Figure 1. Maximum deviation in case of Burgers' equation for $\nu=\frac{1}{100 \pi}$ (top), $\nu=\frac{1}{110 \pi}$ (middle) and $\nu=\frac{1}{120 \pi}$ (bottom).

The KdV equation was numerically solved for the nonlinear parameter values $\alpha=1,6$ with $c=1000$ and $x_{0}=1 / 4$ within the domain $x \in[0,1]$, $t \in\left[0,0.5 \times 10^{-3}\right]$. The initial condition

$$
u_{0}(x)=\frac{3 c}{\alpha} \operatorname{sech}^{2}\left(\frac{1}{2} \sqrt{c}\left(x-x_{0}\right)\right)
$$

and boundary conditions (4.11) were used. In case of HOHWM the additional conditions (4.12) were added. The maximum deviation from the exact solution can be seen in Table 3 .

In case of the KdV equation, the two-soliton exact solution (3.7) was also used. In this case the initial condition was taken from the exact solution as

$$
u_{0}(x)=\frac{3\left(c_{B}-c_{S}\right)\left(c_{B} \operatorname{csch}^{2} \frac{\sqrt{c_{B}}\left(x-x_{0 B}\right)}{2}+c_{S} \operatorname{sech}^{2} \frac{\sqrt{c_{S}}\left(x-x_{0 S}\right)}{2}\right)}{|\alpha|\left(\sqrt{c_{B}} \operatorname{coth} \frac{\sqrt{c_{B}}\left(x-x_{0 B}\right)}{2}-\sqrt{c_{S}} \tanh \frac{\sqrt{c_{S}}\left(x-x_{0 S}\right)}{2}\right)^{2}} .
$$

Boundary conditions (4.11) as well as additional conditions (4.12) were used in this case as well. The results of these calculations can be found in Tables 5 and 6 . 
Table 3. Results for the KdV equation: maximal deviation of the numerical solution from the exact solution $\max \Delta u$ and maximal relative deviation $\max _{r e l} \Delta u$ against the resolution parameter $J$ with initial conditions (5.3) and boundary conditions (4.11) $(\alpha=1, c=1000$, $\left.x_{0}=1 / 4, x \in[0,1], t \in\left[0,0.5 \times 10^{-3}\right]\right)$.

\begin{tabular}{cccccc}
\hline \multirow{2}{*}{$J$} & $2 M$ & \multicolumn{2}{c}{$\max \Delta u$} & \multicolumn{2}{c}{$\max _{\mathrm{rel}} \Delta u$} \\
\cline { 3 - 6 } & & HWM & HOHWM & HWM & HOHWM \\
\hline 4 & 32 & 134.5414 & 32.85357 & 0.0448471 & 0.0109512 \\
5 & 64 & 68.76035 & 3.316314 & 0.0229201 & 0.0011054 \\
6 & 128 & 19.26319 & 0.8202067 & 0.0064211 & 0.0002734 \\
7 & 256 & 5.066037 & 0.844972 & 0.0016887 & 0.0002817 \\
\hline
\end{tabular}

Table 4. Results for the KdV equation: maximal deviation of the numerical solution from the exact solution $\max \Delta u$ and maximal relative deviation $\max _{r e l} \Delta u$ against the resolution parameter $J$ with initial conditions (5.3) and boundary conditions (4.11) ( $\alpha=6, c=1000$, $\left.x_{0}=1 / 4, x \in[0,1], t \in\left[0,0.5 \times 10^{-3}\right]\right)$.

\begin{tabular}{cccccc}
\hline \multirow{2}{*}{$J$} & $2 M$ & \multicolumn{2}{c}{$\max \Delta u$} & \multicolumn{2}{c}{$\max _{\mathrm{rel}} \Delta u$} \\
\cline { 3 - 6 } & & HWM & HOHWM & HWM & HOHWM \\
\hline 4 & 32 & 22.42356 & 5.475594 & 0.0448471 & 0.0109512 \\
5 & 64 & 11.46226 & 0.5527193 & 0.0229245 & 0.0011054 \\
6 & 128 & 3.210801 & 0.1367652 & 0.0064216 & 0.0002735 \\
7 & 256 & 0.8443429 & 0.0016887 & 0.0016887 & 0.0002816 \\
\hline
\end{tabular}

Typical numerical results for the wave equation, the Burgers' equation and the $\mathrm{KdV}$ equation can be seen in Figure 2.

In case of both the Burgers' equation as well as the KdV equation the steep slope can be observed. The soliton interaction and subsequent phase shift can also be observed in case of the $\mathrm{KdV}$ interaction.

Table 5. Results for the KdV equation soliton interaction: maximal deviation of the numerical solution from the exact solution $\max \Delta u$ and maximal relative deviation $\max _{r e l} \Delta u$ against the resolution parameter $J$ with initial conditions (5.4) and boundary conditions (4.11) $\left(\alpha=1, c_{B}=10000, x_{0 B}=1 / 5, c_{S}=10000 / 3, x_{0 S}=2 / 5, x \in[0,1], t \in[0,0.6 \times\right.$ $\left.\left.10^{-4}\right]\right)$.

\begin{tabular}{cccccc}
\hline \multirow{2}{*}{$J$} & $2 M$ & \multicolumn{2}{c}{$\max \Delta u$} & \multicolumn{2}{c}{$\max _{\mathrm{rel}} \Delta u$} \\
\cline { 3 - 6 } & & HWM & HOHWM & HWM & HOHWM \\
\hline 4 & 32 & 3951164 & 2246754 & 131.7054667 & 74.8918000 \\
5 & 64 & 6210848 & 202544.7 & 207.0282667 & 6.7514900 \\
6 & 128 & 1567.924 & 199.7707 & 0.052264 & 0.0066590 \\
7 & 256 & 542.1262 & 14.4892 & 0.0180709 & 0.0004830 \\
\hline
\end{tabular}

Tables $1-6$ show that HOHWM generally gives a more accurate result than HWM. In case of the wave equation (Table 1), the increase in $c_{1}$ (which translates into an increase in the slope) also increases the error of both methods while HOHWM remains more accurate. The Burgers' equation at the values of $\nu$ that were used introduces such a steep slope that at lower resolutions, 

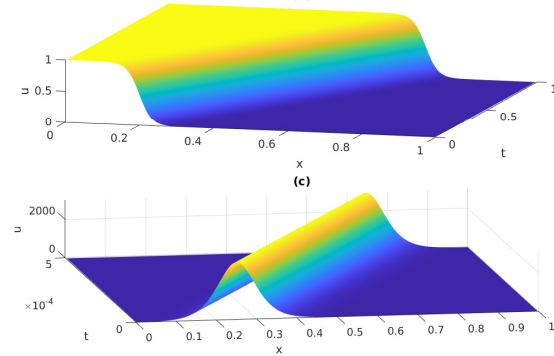

(b)

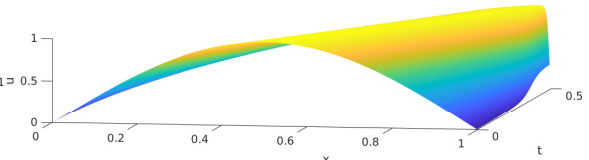

(d)

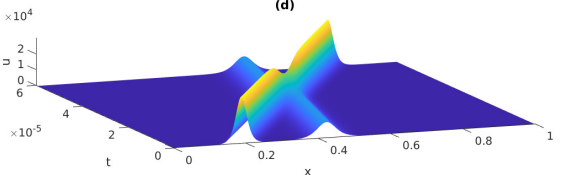

Figure 2. Typical calculated results for HOHWM. (a) Wave equation $\left(c_{1}=60, J=7\right.$ with boundary conditions (4.5) and initial condition (5.1)); (b) Burgers' equation $\left(\nu=\frac{1}{100 \pi}, J=7\right.$ with boundary conditions (4.8) and initial condition (5.2)); (c) KdV single soliton solution $\left(\alpha=1, c=1000, x_{0}=1 / 4, J=7\right.$ with boundary conditions (4.11) and initial conditions (5.3)); (d) Interaction of $\mathrm{KdV}$ solitons $\left(\alpha=1, c_{B}=10000\right.$, $x_{0 B}=1 / 5, c_{S}=10000 / 3, x_{0 S}=2 / 5, J=7$ with boundary conditions (4.11) and initial conditions (5.4)).

Table 6. Results for the KdV equation soliton interaction: maximal deviation of the numerical solution from the exact solution $\max \Delta u$ and maximal relative deviation $\max _{r e l} \Delta u$ against the resolution parameter $J$ with initial conditions (5.4) and boundary conditions (4.11) $\left(\alpha=6, c_{B}=10000, x_{0 B}=1 / 5, c_{S}=10000 / 3, x_{0 S}=2 / 5, x \in[0,1], t \in[0,0.6 \times\right.$ $\left.\left.10^{-4}\right]\right)$.

\begin{tabular}{cccccc}
\hline \multirow{2}{*}{$J$} & \multirow{2}{*}{$2 M$} & \multicolumn{2}{c}{$\max \Delta u$} & \multicolumn{2}{c}{$\max _{\mathrm{rel}} \Delta u$} \\
\cline { 3 - 6 } & & HWM & HOHWM & HWM & HOHWM \\
\hline 4 & 32 & 215719.2 & 380037.1 & 43.1438400 & 76.0074200 \\
5 & 64 & 104514.6 & 31103.69 & 20.9029200 & 6.2207380 \\
6 & 128 & 260.845 & 33.305 & 0.0521690 & 0.0066610 \\
7 & 256 & 90.35663 & 2.415028 & 0.0180713 & 0.0004830 \\
\hline
\end{tabular}

neither method is able to successfully calculate the solution. This is due to the fact that the collocation points are used and at a low resolution, no collocation point falls within the slope. However, it is clear that HOHWM is able to perform calculations further in time before diminishing in accuracy, making it advantageous here as well. Figure 1 shows that HOHWM remains stable for a longer time than HWM since the former does not reach such high error throughout the integration.

In case of the one soliton solutions of the KdV equation, HOHWM again shows a higher accuracy than HWM. It can be noted from Tables 3 and 4 that the HOHWM is more accurate at $J=5(2 M=64)$ than HWM is at $J=7$ $(2 M=256)$.

While the results are not directly comperable with [50] due to the difference in domain as well as values of $c$ and differences in presenting error analysis, some comparison can be made. The maximum relative deviation from the exact solution can be compared. Such a comparison between [50] and results in Table 4 is carried out in Table 7 . While the result with HWM is not as accurate as that of [50] the results with HOHWM surpass the referenced results. 
Table 7. Comparison to [50]: maximal relative deviation from the exact solution at $J=7$.

\begin{tabular}{ccc}
\hline$[50]$ & HWM & HOHWM \\
\hline 0.00041236 & 0.001688031 & 0.0002817978 \\
\hline
\end{tabular}

The KdV soliton interactions show an interesting phenomenon, however. From Tables 5 and 6 one can see that at lower resolutions neither method can successfully solve the problem. This is caused by the fact that one now has two relatively localized solitary waves and at lower resolutions no collocation point falls within these localized solitary waves. However, at higher resolutions, one can clearly see the advantages of HOHWM over HWM as the former shows a significant boost in accuracy.

\section{Conclusions}

The HOHWM has been adapted for solving partial differential equations numerically. The results obtained by the widely used HWM were compared with those obtained by the HOHWM. The Burgers' equation and the KdV equation were considered as model equations.

Our numerical experiments demonstrated that both methods, the HWM and the HOHWM, were able to provide numerical solutions that are in good agreement with the exact analytical solutions. The detailed comparison of the accuracy of the HWM and the HOHWM was performed in Section 5. It is shown that HOHWM can be preferred where high accuracy is required. Furthermore, the HOHWM can also be preferred in cases where high accuracy is not required by applying the method at a lower resolution in comparison with HWM.

Solving the system of algebraic equations is the most computationally expensive task within Haar wavelet based methods. However, the algebraic systems of equations are of the same dimension and have the same symmetric properties for the HWM as well as for the HOHWM. Even though some expressions needed to evaluate are more complex in case of HOHWM, in general it can be concluded that the numerical complexity of the HOHWM is only slightly higher than that of the HWM at the same resolution. It would be more pragmatic to estimate computational complexity of the solution providing the same accuracy, however. For example, in the case of single KdV-soliton solution (Tables 3 and 4), one can use HWM with 256 collocation points or HOHWM with 64 collocation points in order to obtain the same degeree of accuracy. Solving a $64 \times 64$ algebraic system is substantially computationally cheaper than solving a $256 \times 256$ system. Therefore, it is possible to obtain the results with the same accuracy as HWM with lower computational cost by applying HOHWM.

The Fourier transform related pseodospectral method is known as a powerful tool for numerical solution of evolution equations because it is able to produce high accuracy at relatively low number of collocation points [22]. However, 
this method has a disadvantage: because of the nature of the Fourier transform one must apply periodic boundary conditions. Haar wavelet related methods do not have such a disadvantage and one can solve PDEs numerically applying arbitrary boundary conditions in case of the HWM and arbitrary time independent boundary conditions in case of the HOHWM. In order to use time dependent boundary conditions with HOHWM a different numerical scheme must be applied to approximate the temporal derivatives.

\section{Acknowledgements}

This research was supported by the Estonian Research Council (IUT 33-24).

\section{References}

[1] M.J. Ablowitz. Nonlinear evolution equations, inverse scattering and cellular automata. In P.J. Olver and D.H. Sattinger(Eds.), Solitons in Physics, Mathematics, and Nonlinear Optics, pp. 1-26. Springer, 1990.

[2] U. Andersson, B. Engquist, G. Ledfelt and O. Runborg. A contribution to wavelet-based subgrid modeling. Appl. Comput. Harmon. Anal., 7(2):151-164, 1999. https://doi.org/10.1006/acha.1999.0264.

[3] I. Aziz and R. Amin. Numerical solution of a class of delay differential and delay partial differential equations via Haar wavelet. Appl. Math. Model, 40(23):1028610299, 2016. https://doi.org/10.1016/j.apm.2016.07.018.

[4] I. Aziz and I. Khan. Numerical solution of diffusion and reaction-diffusion partial integro-differential equations. Int. J. Comput. Methods, 15(06):1850047, 2018. https://doi.org/10.1016/j.apm.2016.07.018.

[5] I. Aziz and Siraj-ul-Islam. New algorithms for the numerical solution of nonlinear Fredholm and Volterra integral equations using Haar wavelets. Comput. Appl. Math., 239:333-345, 2013. https://doi.org/10.1016/j.cam.2012.08.031.

[6] I. Aziz, Siraj-ul-Islam and M. Asif. Haar wavelet collocation method for three-dimensional elliptic partial differential equations. Comput. Math. Appl., 73(9):2023-2034, 2017. https://doi.org/10.1016/j.camwa.2017.02.034.

[7] I. Aziz, Siraj-ul-Islam and F. Khan. A new method based on Haar wavelet for the numerical solution of two-dimensional nonlinear integral equations. Comput. Appl. Math., 272:70-80, 2014. https://doi.org/10.1016/j.cam.2014.04.027.

[8] E. Babolian and A. Shahsavaran. Numerical solution of nonlinear Fredholm integral equations of the second kind using Haar wavelets. Comput. Appl. Math., 225(1):87-95, 2009. https://doi.org/10.1016/j.cam.2008.07.003.

[9] E.R. Benton and G.W. Platzman. A table of solutions of the onedimensional Burgers equation. Quart. Appl. Math., 30(2):195-212, 1972. https://doi.org/10.1090/qam/306736.

[10] J.M. Burgers. A mathematical model illustrating the theory of turbulence. In Richard Von Mises and Theodore Von Kármán(Eds.), Advances in Applied Mechanics, volume 1, pp. 171-199. Academic Press, 1948. https://doi.org/10.1016/S0065-2156(08)70100-5.

[11] J.M. Burgers. Statistical problems connected with the solution of a nonlinear partial differential equation. In William F. Ames(Ed.), Nonlinear Problems of 
Engineering, pp. 123-137. Academic Press, 1964. https://doi.org/10.1016/B9781-4832-0078-1.50015-8.

[12] C. Cattani. Haar wavelets based technique in evolution problems. Proc. Estonian Acad. Sci. Phys. Math., 53(1):45-63, 2004.

[13] C. Cattani. Harmonic wavelets towards the solution of nonlinear PDE. Comput. Math. Appl., 50(8-9):1191-1210, 2005. https://doi.org/10.1016/j.camwa.2005.07.001.

[14] C. Cattani and A. Kudreyko. Harmonic wavelet method towards solution of the Fredholm type integral equations of the second kind. Appl. Math. Comput., 215(12):4164-4171, 2010. https://doi.org/10.1016/j.amc.2009.12.037.

[15] C.F. Chen and C. H. Hsiao. Haar wavelet method for solving lumped and distributed-parameter systems. IEE Proc. Control Theory Appl., 144(1):87-94, 1997. https://doi.org/10.1049/ip-cta:19970702.

[16] J.D. Cole. On a quasi-linear parabolic equation occurring in aerodynamics. Quart. Appl. Math., 9(3):225-236, 1951. https://doi.org/10.1090/qam/42889.

[17] J.R. Dormand and P.J. Prince. A family of embedded Runge-Kutta formulae. J. Comput. Appl. Math., 6(1):19-26, 1980. https://doi.org/10.1016/0771050X(80)90013-3.

[18] P.G. Drazin. Solitons, volume 85 of London Mathematical Society Lecture Note Series. Cambridge University Press, 1983.

[19] P.G. Drazin and R.S. Johnson. Solitons: an introduction. Cambridge University Press, 1989. https://doi.org/10.1002/zamm.19900700817.

[20] J. Engelbrecht and A. Salupere. On the problem of periodicity and hidden solitons for the KdV model. Chaos, 15:015114, 2005. https://doi.org/10.1063/1.1858781.

[21] S. Foadian, R. Pourgholi, S.H. Tabasi and J. Damirchi. The inverse solution of the coupled nonlinear reaction-diffusion equations by the Haar wavelets. Int. J. Comput. Math., 96(1):105-125, 2019. https://doi.org/10.1080/00207160.2017.1417593.

[22] B. Fornberg. A Practical Guide to Pseudospectral Methods. Cambridge University Press, Cambridge, 1998. https://doi.org/10.1017/CBO9780511626357.

[23] Y. Ge, S. Li, Y. Shi and L. Han. An adaptive wavelet method for solving mixedinteger dynamic optimization problems with discontinuous controls and application to alkali-surfactant-polymer flooding. Eng. Optim., 51(6):1028-1048, 2019. https://doi.org/10.1080/0305215X.2018.1508573.

[24] M.F. Hamilton and D. T. Blackstock(Eds.). Nonlinear acoustics. Academic Press, San Diego, 1998.

[25] G. Hariharan, K. Kannan and K.R. Sharma. Haar wavelet method for solving fishers equation. Appl. Math. Comput., 211(2):284-292, 2009. https://doi.org/10.1016/j.amc.2008.12.089.

[26] H. Hein and L. Feklistova. Computationally efficient delamination detection in composite beams using Haar wavelets. Mech. Syst. Signal Process., 25(6):22572270, 2011.

[27] C.H. Hsiao. State analysis of linear time delayed systems via Haar wavelets. Math. Comput. Simulation, 44(5):457-470, 1997. https://doi.org/10.1016/S0378-4754(97)00075-X. 
[28] G. Jin, X. Xie and Z. Liu. The Haar wavelet method for free vibration analysis of functionally graded cylindrical shells based on the shear deformation theory. Compos. Struct., 108:435-448, 2014. https://doi.org/10.1016/j.compstruct.2013.09.044.

[29] R. Jiwari. A Haar wavelet quasilinearization approach for numerical simulation of Burgers equation. Comput. Phys. Comm., 183(11):2413-2423, 2012. https://doi.org/10.1016/j.cpc.2012.06.009.

[30] M. Kirs, K. Karjust, I. Aziz, E. Õunapuu and E. Tungel. Free vibration analysis of a functionally graded material beam: evaluation of the Haar wavelet method. Proc. Estonian Acad. Sci., 67(1):1-9, 2018.

[31] P.A. Lagerstrom, J.D. Cole and L. Trilling. Problems in the theory of viscous compressible fluids. Guggenheim Aeronautical Laboratory, unnumbered report, California Institute of Technology, 1949.

[32] G. L. Lamb Jr. Elements of Soliton Theory. John Wiley \& Sons, 1980.

[33] Ü. Lepik. Numerical solution of differential equations using Haar wavelets. Math. Comput. Simulation, 68(2):127-143, 2005. https://doi.org/10.1016/j.matcom.2004.10.005.

[34] Ü. Lepik. Haar wavelet method for nonlinear integro-differential equations. Appl. Math. Comput., 176(1):324-333, 2006. https://doi.org/10.1016/j.amc.2005.09.021.

[35] Ü. Lepik. Application of the Haar wavelet transform to solving integral and differential equations. Proc. Estonian Acad. Sci. Phys. Math., 56(1):28-46, 2007. https://doi.org/10.1117/12.736416.

[36] Ü. Lepik. Numerical solution of evolution equations by the Haar wavelet method. Appl. Math. Comput., 185(1):695-704, 2007. https://doi.org/10.1016/j.amc.2006.07.077.

[37] Ü. Lepik. Solving fractional integral equations by the Haar wavelet method. Appl. Math. Comput., 214(2):468-478, 2009. https://doi.org/10.1016/j.amc.2009.04.015.

[38] Ü. Lepik. Solving PDEs with the aid of two-dimensional Haar wavelets. Comput. Math. Appl., 61(7):1873-1879, 2011. https://doi.org/10.1016/j.camwa.2011.02.016.

[39] Ü. Lepik and H. Hein. Haar wavelets: with applications. Springer, 2014. https://doi.org/10.1007/978-3-319-04295-4.

[40] J. Majak, M. Pohlak and M. Eerme. Application of the Haar wavelet-based discretization technique to problems of orthotropic plates and shells. Mech. Compos. Mater., 45(6):631-642, 2009. https://doi.org/10.1007/s11029-010-9119-0.

[41] J. Majak, M. Pohlak, M. Eerme and T. Lepikult. Weak formulation based Haar wavelet method for solving differential equations. Appl. Math. Comput., 211(2):488-494, 2009. https://doi.org/10.1016/j.amc.2009.01.089.

[42] J. Majak, M. Pohlak, K. Karjust, M. Eerme, J. Kurnitski and B.S. Shvartsman. New higher order Haar wavelet method: Application to FGM structures. Compos. Struct., 201:72-78, 2018. https://doi.org/10.1016/j.compstruct.2018.06.013.

[43] J. Majak, B. Shvartsman, K. Karjust, M. Mikola, A. Haavaj oe and M. Pohlak. On the accuracy of the Haar wavelet discretization method. Compos. Part B, 80:321-327, 2015. https://doi.org/10.1016/j.compositesb.2015.06.008. 
[44] J. Majak, B. Shvartsman, M. Pohlak, K. Karjust, M. Eerme and E. Tungel. Solution of fractional order differential equation by the Haar Wavelet method. numerical convergence analysis for most commonly used approach. AIP Conference Proceedings, 1738(1):480110, 2016. https://doi.org/10.1063/1.4952346.

[45] J. Majak, B.S. Shvartsman, M. Kirs, M. Pohlak and H. Herranen. Convergence theorem for the Haar wavelet based discretization method. Compos. Struct., 126:227-232, 2015. https://doi.org/10.1016/j.compstruct.2015.02.050.

[46] T. Musha and H. Higuchi. Traffic current fluctuation and the Burgers equation. Jpn. J. Appl. Phys., 17(5):811-816, 1978. https://doi.org/10.1143/JJAP.17.811.

[47] S. Nazir, S. Shahzad, R. Wirza, R. Amin, M. Ahsan, N. Mukhtar, Iván. García-Magariño and J. Lloret. Birthmark based identification of software piracy using Haar wavelet. Math. Comput. Simulation, 2019. https://doi.org/10.1016/j.matcom.2019.04.010.

[48] Ö. Oruç. A non-uniform Haar wavelet method for numerically solving two-dimensional convection-dominated equations and two-dimensional near singular elliptic equations. Comput. Math. Appl., 77(7):1799-1820, 2019. https://doi.org/10.1016/j.camwa.2018.11.018.

[49] Ö. Oruç, F. Bulut and A. Esen. A Haar wavelet-finite difference hybrid method for the numerical solution of the modified Burgers equation. J. Math. Chem., 53(7):1592-1607, 2015. https://doi.org/10.1007/s10910-015-0507-5.

[50] Ö. Oruç, F. Bulut and A. Esen. Numerical solution of the KdV equation by Haar wavelet method. Pramana - J. Phys., 87(6):94, Nov 2016. https://doi.org/10.1007/s12043-016-1286-7.

[51] Ö. Oruç, F. Bulut and A. Esen. Numerical solutions of regularized long wave equation by Haar wavelet method. Mediterr. J. Math., 13(5):3235-3253, 2016. https://doi.org/10.1007/s00009-016-0682-z.

[52] Ö. Oruç, F. Bulut and A. Esen. A numerical treatment based on Haar wavelets for coupled KdV equation. Int. J. Optim. Control, 7(2):195-204, 2017. https://doi.org/10.11121/ijocta.01.2017.00396.

[53] Ö. Oruç, A. Esen and F. Bulut. A Haar wavelet approximation for twodimensional time fractional reaction-subdiffusion equation. Eng. Comput., 35(1):75-86, Jan 2019. ISSN 1435-5663. https://doi.org/10.1007/s00366-0180584-8.

[54] M. Remoissenet. Waves called solitons: concepts and experiments. Springer, 2013.

[55] A. Salupere. The pseudospectral method and discrete spectral analysis. In Ewald Quak and Tarmo Soomere(Eds.), Applied Wave Mathematics: Selected Topics in Solids, Fluids, and Mathematical Methods, pp. 301-333. Springer, 2009.

[56] A. Salupere, J. Engelbrecht, O. Ilison and L. Ilison. On solitons in microstructured solids and granular materials. Math. Comput. Simulation, 69:502-513, 2005. https://doi.org/10.1016/j.matcom.2005.03.015.

[57] A. Salupere, P. Peterson and J. Engelbrecht. Long-time behaviour of soliton ensembles. Part I-Emergence of ensembles. Chaos Solitons Fractals, 14(9):14131424, 2002. https://doi.org/10.1016/S0960-0779(02)00069-3.

[58] A. Salupere and M. Ratas. On the application of 2D discrete spectral analysis in case of the kp equation. Mech. Research Comm., 93:141- 147, 2018. https://doi.org/10.1016/j.mechrescom.2017.08.010. 
[59] I. Sertakov, J. Engelbrecht and J. Janno. Modelling 2D wave motion in microstructured solids. Mech. Research Comm., 56:42 - 49, 2014. https://doi.org/10.1016/j.mechrescom.2013.11.007.

[60] A. Setia, B. Prakash and A.S. Vatsala. Haar based numerical solution of Fredholm-Volterra fractional integro-differential equation with nonlocal boundary conditions. In AIP Conference Proceedings, volume 1798, p. 020140. AIP Publishing, 2017. https://doi.org/10.1063/1.4972732.

[61] L.F. Shampine and M.W. Reichelt. The MATLAB ODE suite. SIAM J. Sci. Comput., 18(1):1-22, 1997. https://doi.org/10.1137/S1064827594276424.

[62] X. Si, C. Wang, Y. Shen and L. Zheng. Numerical method to initialboundary value problems for fractional partial differential equations with time-space variable coefficients. Appl. Math. Model, 40(7-8):4397-4411, 2016. https://doi.org/10.1016/j.apm.2015.11.039.

[63] Siraj-ul-Islam, I. Aziz and A.S. Al-Fhaid. An improved method based on Haar wavelets for numerical solution of nonlinear integral and integro-differential equations of first and higher orders. Comput. Appl. Math., 260:449-469, 2014. https://doi.org/10.1016/j.cam.2013.10.024.

[64] T.J. Wang and T. Sun. Mixed pseudospectral method for heat transfer. Math. Mod. Anal., 21(2):199-219, 2016. https://doi.org/10.3846/13926292.2016.1146925.

[65] N. Wichailukkanaa, B. Novaprateepa and C. Boonyasiriwatc. A convergence analysis of the numerical solution of boundary-value problems by using two-dimensional Haar wavelets. ScienceAsia, 42(5):346-355, 2016. https://doi.org/10.2306/scienceasia1513-1874.2016.42.346.

[66] X. Xiang, J. Guoyong, L. Wanyou and L. Zhigang. A numerical solution for vibration analysis of composite laminated conical, cylindrical shell and annular plate structures. Compos. Struct., 111:20-30, 2014. https://doi.org/10.1016/j.compstruct.2013.12.019.

[67] X. Xie, G. Jin and Z. Liu. Free vibration analysis of cylindrical shells using the Haar wavelet method. Int. J. Mech. Sci., 77:47-56, 2013. https://doi.org/10.1016/j.ijmecsci.2013.09.025.

[68] X. Xie, G. Jin, Y. Yan, S.X. Shi and Z. Liu. Free vibration analysis of composite laminated cylindrical shells using the Haar wavelet method. Compos. Struct., 109:169-177, 2014. https://doi.org/10.1016/j.compstruct.2013.10.058.

[69] X. Xie, G. Jin, T. Ye and Z. Liu. Free vibration analysis of functionally graded conical shells and annular plates using the Haar wavelet method. Appl. Acoust., 85:130-142, 2014. https://doi.org/10.1016/j.apacoust.2014.04.006.

[70] T. Yoneyama. The Korteweg-de Vries two-soliton solution as interacting two single solitons. Progr. Theoret. Phys., 71(4):843-846, 1984. https://doi.org/10.1143/PTP.71.843. 